\title{
Extratos aquosos de Leucaena leucocephala e Sterculia foetida no controle de Bemisia tabaci biótipo B (Hemiptera: Aleyrodidae)
}

\author{
Aqueous extracts of Leucaena leucocephala and Sterculia foetida to the control of Bemisia \\ tabaci biotype B (Hemiptera: Aleyrodidae)
}

\author{
Geraldo José Nascimento de Vasconcelos ${ }^{1}$ Manoel Guedes Corrêa Gondim Júnior ${ }^{2}$ \\ Reginaldo Barros ${ }^{2}$
}

\section{RESUMO}

A presente pesquisa foi desenvolvida na Cidade de Recife-PE, com o objetivo de avaliar a ação inseticida insetistática e repelente de extratos aquosos de duas essências florestais sobre ovos, ninfas e adultos de Bemisia tabaci biótipo B (Genn., 1889) criados em Brassica oleracea var. acephala L. Os extratos utilizados foram de Leucaena leucocephala (Lam.) De Wit. e Sterculia foetida L. Sementes e folhas das duas espécies foram coletadas, secadas e trituradas. Em seguida, os extratos foram preparados na proporção de $5 \mathrm{~g}$ de cada parte vegetal/50mL de água. Folhas de B. oleracea var. acephala foram imersas durante $30 \mathrm{seg}$ nos extratos e, em seguida, os insetos foram confinados na folha tratada. Os extratos de sementes de $\mathbf{L}$. leucocephala e de folhas de $\boldsymbol{S}$. foetida provocaram mortalidade de 60 e $41 \%$ sobre ovos de $\boldsymbol{B}$. tabaci, respectivamente. Na fase ninfal, o tratamento com folhas de $S$. foetida apresentou o melhor resultado, com $74,6 \%$ de mortalidade. Nenhum dos extratos testados apresentou supressão para oviposição na espécie estudada. Os indivíduos tratados com os extratos de L. leucocephala tiveram menor longevidade e maior oviposição com relação aos tratamentos com $\boldsymbol{S}$. foetida e com a testemunha. $\mathrm{O}$ uso de extratos aquosos de sementes de L. leucocephala e de folhas de $\boldsymbol{S}$. foetida mostrase promissor para o controle alternativo de B. tabaci.

Palavras-chave: horticultura, mosca-branca, planta inseticida, Mimosidae, Sterculiaceae.

\section{ABSTRACT}

The present research was carried out in Recife-PE, aimed at to evaluating insecticide, insectistatic and repellant effects of aqueous extracts of Leucaena leucocephala (Lam.) De Wit. and Sterculia foetida L. on eggs, nymphs and adults of Bemisia tabaci biotype B (Genn., 1889) on Brassica oleracea var. acephala L. culture. Seeds and leaves of L. leucocephala and $\mathbf{S}$. foetida were collected, dried and ground. Solutions from the aqueous extracts were prepared with $5 \mathrm{~g}$ of dried vegetable and $50 \mathrm{ml}$ of pure water. Leaves of B. oleracea var. acephala were sunk in the solutions for 30 seconds before confining insects on them. Extracts of L. leucocephala seeds and $\boldsymbol{S}$. foetida leaves caused mortality of $60 \%$ and $41 \%$, respectively, on eggs of $\boldsymbol{B}$. tabaci. Leaves of $\boldsymbol{S}$. foetida caused the highest effects on nymphal instars, with mortality of $74.6 \%$. None of the tested extract showed suppression on insect oviposition. The treatments of $\boldsymbol{L}$. leucocephala showed a shorter longevity and higher oviposition than the treatments of $\boldsymbol{S}$. foetida and the control. The aqueous extracts from $\mathbf{L}$. leucocephala seeds and $\boldsymbol{S}$. foetida leaves can be a good alternative to the control of B. tabaci.

Key words: horticultural-crops, whitefly, insecticidal plant, Mimosidae, Sterculiaceae.

\section{INTRODUÇÃO}

A mosca-branca, Bemisia tabaci biótipo B (Genn., 1889), é uma das principais pragas da agricultura mundial, tanto pela sua agressividade como pela diversidade de hospedeiros que apresenta (VILLAS BÔAS et al., 1997; OLIVEIRA et al., 2001). Além do alto potencial de causar danos, esse inseto também é considerado o mais importante vetor de fitoviroses em todo o mundo (GERLING, 2000). O manejo da moscabranca tornou-se um grande desafio, pois a dispersão entre as culturas, o alto potencial reprodutivo e o hábito polífago contribuem para a complexidade e a dificuldade de controle (TOSCANO et al., 2003). Na tentativa de controlar a infestação dessa praga, tem-se adotado

${ }^{1}$ Departamento de Entomologia Fitopatologia e Zoologia Agrícola, Escola Superior de Agricultura "Luiz de Queiroz”, Universidade de São Paulo (ESALQ, USP). Avenida Pádua Dias, 11, CP 9, 13418-900, Piracicaba, São Paulo, Brasil. E-mail: gvasconcelosagro@hotmail.com. Autor para correspondência.

${ }^{2}$ Departamento de Agronomia/Fitossanidade, Universidade Federal Rural de Pernambuco (UFRPE), Recife, Pernambuco, Brasil 
várias medidas, como a utilização de inseticidas sintéticos, métodos de controle cultural, biológico e resistência de plantas, isoladamente ou em conjunto, no manejo integrado (HOROWITZ \& ISHAAYA, 1995; SUJII et al., 2002; AZEVEDO et al., 2005; BALDIN et al., 2005).

Uma alternativa que vem sendo retomada atualmente para o controle de pragas é o uso de substâncias secundárias presentes em "plantas inseticidas”. Diversas substâncias oriundas dos produtos intermediários ou finais do metabolismo secundário de plantas, que podem ser encontradas principalmente em suas partes vitais, tais como raízes, folhas e sementes (MEDEIROS, 1990), sobretudo rotenóides, piretróides, alcalóides e terpenóides, podem interferir severamente no metabolismo de outros organismos, causando impactos variáveis, como repelência, deterrência alimentar e de oviposição, esterilização, bloqueio do metabolismo e interferência no desenvolvimento, sem necessariamente causar morte (LANCHER, 2000). Neste último caso, pode haver retardamento no desenvolvimento do inseto, causando, segundo HERNANDEZ \& VEDRAMIM (1998), efeito insetistático. Vários extratos de plantas já foram testados sobre $\boldsymbol{B}$. tabaci, promovendo diversos efeitos, desde a repelência para oviposição até a mortalidade nas diversas fases do seu ciclo biológico (SOUZA, 2004). O uso de extratos vegetais no controle de $\boldsymbol{B}$. tabaci envolve avaliação de diversas partes vegetais, de diferentes espécies, sobretudo da família Meliaceae (NARDO et al., 1997; HAMMAD et al., 2000; SOUZA \& VENDRAMIM, 2000; SILVA et al., 2003; SOUZA, 2004).

Além das Meliaceae, outras espécies botânicas das famílias Annonaceae, Asteraceae, Cannellaceae, Labiateae e Rutaceae também são promissoras no controle de pragas (JACOBSON, 1989). Entretanto, ainda são escassos os estudos sobre o potencial inseticida para a grande maioria das espécies vegetais, sendo de grande relevância o desenvolvimento de pesquisas nesta área, em busca de novas alternativas.

A leucena (Leucaena leucocephala (Lam.) De Wit.) é uma Leguminosae (Mimosidae) originária do México, sendo encontrada em toda a região tropical (SKERMAN, 1977). Essa planta apresenta múltipla utilização, destacando-se o reflorestamento de áreas degradadas, a alimentação animal e a adubação verde (PRATES et al., 2000). O chichá (Sterculia foetida L.) é uma árvore da família Sterculiaceae, originária da Ásia, que foi introduzida no Brasil como árvore ornamental e de sombra. Atualmente, tem sido indicada para recomposição de áreas degradadas (SANTOS et al., 2004).
Este trabalho teve como objetivo avaliar a ação inseticida e insetistática sobre ovos, ninfas e adultos e supressão de oviposição de extrato aquoso de $\mathbf{L}$. leucocephala e $\boldsymbol{S}$. foetida sobre $\boldsymbol{B}$. tabaci.

\section{MATERIAL E MÉTODOS}

A pesquisa foi desenvolvida na Cidade de Recife, na Universidade Federal Rural de Pernambuco, onde foram desenvolvidas as seguintes etapas:

\section{Obtenção de mudas}

Sementes de couve, Brassica oleracea var. acephala L., foram semeadas em bandejas de isopor contendo substrato Plant Max Hortaliças HA ${ }^{\circledR}$, em casa de vegetação. Após 30 dias, as mudas foram transplantas para vasos plásticos de $2.500 \mathrm{~cm}^{3}$ contendo solo esterilizado. Os tratos culturais adotados foram os padrões para a cultura (FILGUEIRA, 2000).

Obtenção de extratos

Foram coletadas folhas verdes e sementes secas de $\boldsymbol{L}$. leucocephala e de $\boldsymbol{S}$. foetida em áreas do Campus da UFRPE. Todo o material coletado foi secado em estufa a $50^{\circ} \mathrm{C}$ por $48 \mathrm{~h}$ e, em seguida, foram triturados, em liquidificador, e armazenados em recipientes hermeticamente fechados (TAVARES, 2002).

Os extratos, na concentração de $10 \%$ (peso/ volume), foram obtidos pela adição de $5 \mathrm{~g}$ de pó a $50 \mathrm{~mL}$ de água e mantidos, por 24h, em frascos fechados para extração das substâncias secundárias hidrossolúveis, sobretudo terpenóides glicosados, que possuem ampla distribuição no reino vegetal, e que são caracterizados por sua elevada ação tóxica e solubilidade em água (LIMA, 2000). Em seguida, as soluções foram filtradas com tecido tipo voil.

\section{Criação de $\boldsymbol{B}$. tabaci}

A criação deste inseto foi estabelecida utilizando como hospedeiro B. oleracea var. acephala cultivada em casa de vegetação, onde a espécie em estudo ocorre naturalmente. Após a infestação do hospedeiro, as bancadas utilizadas foram teladas para evitar a fuga dos adultos. Para confirmação da espécie, algumas amostras foram montadas e identificadas de acordo com MARTIN (1987). As médias de temperatura e umidade relativa durante o período em que a criação foi mantida na casa de vegetação foram $28,5 \pm 2,16^{\circ} \mathrm{C}$ e $72 \pm 10,2 \%$, respectivamente.

\section{Efeito no período embrionário}

Foram confinados 50 adultos por repetição, não-sexados, em gaiolas confeccionadas com tecido 
tipo voil, por 24h, para obtenção dos ovos. Ao término do confinamento, foram selecionadas folhas com, no mínimo, 50 ovos. As folhas selecionadas foram destacadas na base do pecíolo, sendo este envolto por algodão umedecido. Posteriormente, o limbo foliar foi imerso durante 30seg no extrato correspondente ao tratamento e posto para secar à sombra por $30 \mathrm{~min}$. A testemunha foi imersa em água destilada durante $30 \mathrm{seg}$ e posta para secar à sombra por $30 \mathrm{~min}$. Por fim, cada folha foi sobreposta em um papel de filtro, o qual foi colocado sobre uma espuma de polietileno umedecido com água, que se encontrava no interior de uma placa de Petri. As avaliações foram feitas diariamente, observando-se a duração do estágio embrionário. Após não se verificar mais a eclosão de ninfas, foi determinada a mortalidade da fase, baseando-se no número inicial de ovos e no número de ninfa que eclodiram.

Efeito na fase ninfal

Foram colocadas plantas de B. oleracea var. acephala, isentas de $\boldsymbol{B}$. tabaci, na bancada telada utilizada para criação por $24 \mathrm{~h}$ para obtenção de ovos. Após este período, foram selecionadas duas folhas por planta, com no mínimo 50 ovos, sendo as demais eliminadas. Três dias após a eclosão das primeiras ninfas, foi contabilizado o número de ninfa/folha e, em seguida, foi feita a imersão das folhas, da mesma forma que o experimento anterior. As avaliações foram feitas diariamente, observando-se a duração do estágio ninfal. As plantas utilizadas foram mantidas isoladas em gaiola de tecido tipo voil, com $15 \mathrm{~cm}$ de diâmetro por $30 \mathrm{~cm}$ de altura. Após a emergência de todos os adultos, foi determinada a mortalidade da fase, baseando-se no número inicial de ninfas e no número de adultos que emergiram.

Índice de supressão de oviposição

Para determinar a supressão de oviposição, foram selecionadas plantas de $\boldsymbol{B}$. oleracea var. acephala, de mesma idade fisiológica, nas quais foram feitos desbastes deixando-se apenas a $4^{\mathrm{a}}$ e a $5^{\mathrm{a}}$ folha emitidas. Em seguida, uma das folhas foi imersa, durante 30seg, em um dos extratos, e a outra em água destilada. Posteriormente, as plantas foram postas para secar à sombra, durante 30min. Após a secagem, as plantas foram individualizadas em uma gaiola de tecido tipo voil, com $15 \mathrm{~cm}$ de diâmetro por $30 \mathrm{~cm}$ de altura. Por fim, foram liberados 15 adultos de $\boldsymbol{B}$. tabaci, não sexados, no interior de cada gaiola. Os insetos utilizados nos testes tinham entre 0 a 24 h de idade.
Após 48h, foram retirados os adultos e no laboratório, procedeu-se a contagem do número de ovos, com auxílio de microscópio estereoscópico. Posteriormente, calculou-se o Índice de Supressão de Oviposição, por meio de uma adaptação da expressão sugerida por KOGAN \& GOEDEN (1970): ISO = 2A/(M + A), em que: ISO = índice de Supressão de Oviposição; $\mathrm{A}=$ quantidade de ovos na folha tratada com extrato vegetal e $\mathrm{M}$ = quantidade de ovos na folha tratada com água. Os valores de ISO variam entre zero e dois, indicando: $\mathrm{ISO}=1$, tratamento neutro; ISO $>1$, oviposição favorecida e ISO $<1$, oviposição suprimida.

Como margem de segurança para essa classificação, o desvio padrão (DP) de cada tratamento foi adicionado ou subtraído do valor 1,00 (indicativo de neutralidade), de forma que cada tratamento só foi considerado favorável ou supressivo para oviposição quando o ISO estava fora do intervalo 1,00 \pm DP.

Longevidade e fertilidade

Para avaliar a longevidade e a fecundidade de $\boldsymbol{B}$. tabaci, foram confinados grupos de quinze indivíduos, não sexados, com 0 a 24h de idade, em uma gaiola de acrílico, $10 \mathrm{~cm}$ de altura por $5 \mathrm{~cm}$ de diâmetro, com a parte superior coberta com tecido tipo voil para permitir a aeração. Em cada gaiola, foi colocada uma folha tratada, a qual continuou ligada à planta. Durante o decorrer do experimento, as folhas foram, trocadas a cada quinze dias, por outras com o mesmo tratamento e ligadas à planta de origem.

Diariamente, foi anotada a mortalidade dos adultos, ocasião em que eram retirados e sexados. A sexagem foi feita com auxílio de microscópioestereoscópico, sendo o reconhecimento dos machos e fêmeas feito por meio do tamanho dos adultos e das diferenças anatômicas do abdômen do inseto. A observação de cada gaiola foi considerada concluída com a morte de todos os indivíduos. A contagem dos ovos de cada folha foi feita na ocasião da troca do alimento.

\section{Análise estatística}

Foi utilizado o delineamento inteiramente casualizado, com quatro tratamentos e dez repetições, para o experimento de preferência de oviposição, e cinco tratamentos com sete repetições, para os demais experimentos. Todos os resultados foram submetidos à análise de variância, e as médias foram comparadas pelo teste de Tukey a 5\% de probabilidade de erro. Nos experimentos de preferência de oviposição, de longevidade e de fecundidade, os dados foram transformados em $(\mathrm{x}+0,5)^{1 / 2}$. 


\section{RESULTADOS E DISCUSSÃO}

Efeito no período embrionário

A duração do período embrionário foi significativamente maior, em relação à testemunha (5,9 dias), nos tratamentos com extratos de sementes de $\boldsymbol{L}$. leucocephala (6,8 dias) e de folhas de $\boldsymbol{S}$. foetida (6,7 dias), não havendo diferença significativa entre estes dois tratamentos, pressupondo uma ação insetistática destes extratos sobre B. tabaci (Tabela 1). É provável que a diferença no efeito proporcionado por diferentes estruturas da mesma planta seja devida ao fato de que os componentes secundários não estão distribuídos uniformemente por todos os órgãos da planta, podendo provocar diferença na toxicidade do extrato oriundo destas diferentes estruturas. SOUZA \& VENDRAMIM (2000), estudando a ação de extratos aquosos em $\boldsymbol{B}$. tabaci biótipo B a 3\% de sementes de Azadirachta indica A. Juss, frutos verdes de Melia azedarach L. e ramos de Trichilia pallida Swartz, obtiveram duração do período embrionário próximas às observadas para os extratos de sementes de $\mathbf{L}$. leucocephala e para as folhas de $\boldsymbol{S}$. foetida; no entanto, estes autores não constataram prolongamento do período em relação à testemunha. CAVALCANTE (2004), estudando a ação de extratos aquosos $10 \%$ de folhas de $\boldsymbol{A}$. indica, Prosopis juliflora (Sw) DC., L. leucocephala, Eucalyptus citriodora Hook, Mimosa caesalpiniaefolia Benth e Myracrodruon urundeuva Fr. All., sobre B. tabaci biótipo B, também obteve resultados similares.

Os tratamentos com sementes de $\boldsymbol{L}$. leucocephala e com folhas de S. foetida apresentaram as maiores taxas de mortalidade quando aplicados sobre ovos de B. tabaci, ocasionando, respectivamente, mortalidade de 60 e $41 \%$, sendo estatisticamente diferentes dos demais tratamentos, os quais não diferiram entre si e apresentaram uma taxa de mortalidade que variou de 7 a 11\% (Tabela 1). Igualmente como o observado na duração do período embrionário, os extratos produzidos com estas duas estruturas vegetais aparentam conter uma maior concentração da provável substância, ou complexo de substâncias, respondível pela toxicidade do extrato, refletindo em uma maior mortalidade sobre os ovos. As mortalidades ocasionadas por estes dois extratos são superiores às registradas por SOUZA \& VENDRAMIM (2000), que foi de $31,3 \%$; $28,9 \%$ e $38,7 \%$, quando aplicaram, respectivamente, extratos a $3 \%$ de sementes de $\boldsymbol{A}$. indica, frutos verdes de $\boldsymbol{M}$. azedarach e ramos de T. pallida, sobre ovos de B. tabaci. CAVALCANTE (2004), estudando este mesmo inseto, relatou percentagens de mortalidade de $56,4 \%$; 43,6\% e 42,0\%, para extratos aquosos a $10 \%$ de folhas de $\boldsymbol{A}$. indica, $\boldsymbol{P}$. juliflora e L. leucocephala, respectivamente.

\section{Efeito na fase ninfal}

A duração do estágio ninfal não apresentou diferença entre a testemunha e os extratos testados, com médias variando entre 13,2 e 13,9 dias (Tabela 1). SOUZA \& VENDRAMIM (2000); CAVALCANTE (2004) também não observaram alteração no período de duração da fase ninfal de B. tabaci (aproximadamente 13 dias) quando submetidas a tratamentos com diversos extratos vegetais, com concentrações variando de 3 a 10\%.

Tabela 1 - Duração e mortalidade das fases de ovo e ninfa (médias \pm EP) de Bemisia tabaci biótipo B, tratadas com extratos aquosos a 10\% de Leucaena leucocephala e Sterculia foetida.

\begin{tabular}{|c|c|c|c|c|c|}
\hline & & \multicolumn{4}{|c|}{ Estágio } \\
\hline \multicolumn{2}{|c|}{ Tratamento $^{1}$} & \multicolumn{2}{|c|}{ Ovo } & \multicolumn{2}{|c|}{ Ninfa } \\
\hline & & Duração (dias) & Mortalidade (\%) & Duração (dias) & Mortalidade (\%) \\
\hline \multirow[t]{2}{*}{ L. leucocephala } & Folha & $6,3 \pm 0,12 \mathrm{bc}^{2}$ & $10,6 \pm 3,97 \mathrm{~b}$ & $13,5 \pm 0,04 \mathrm{a}$ & $22,1 \pm 5,62 \mathrm{~b}$ \\
\hline & Semente & $6,8 \pm 0,15$ a & $60,0 \pm 6,09$ a & $13,2 \pm 0,15 \mathrm{a}$ & $17,5 \pm 5,94 \mathrm{~b}$ \\
\hline \multirow[t]{2}{*}{ S. foetida } & Folha & $6,7 \pm 0,12 \mathrm{ab}$ & $41,4 \pm 7,44$ a & $13,9 \pm 0,32 \mathrm{a}$ & $74,6 \pm 2,44 \mathrm{a}$ \\
\hline & Semente & $5,9 \pm 0,06 \mathrm{c}$ & $8,0 \pm 2,31 \mathrm{~b}$ & $13,5 \pm 0,19 \mathrm{a}$ & $27,5 \pm 7,83 \mathrm{~b}$ \\
\hline Testemunha & Água & $5,9 \pm 0,05 \mathrm{c}$ & $7,2 \pm 3,90 \mathrm{~b}$ & $13,4 \pm 0,13$ a & $7,5 \pm 2,78 \mathrm{~b}$ \\
\hline Média geral & -- & $6,3 \pm 0,10$ & $25,4 \pm 4,72$ & $13,5 \pm 0,17$ & $29,8 \pm 4,92$ \\
\hline CV (\%) & -- & 4,6 & 18,0 & 3,7 & 20,1 \\
\hline
\end{tabular}

${ }^{1}$ Temperatura média: $28,3 \pm 2,9^{\circ} \mathrm{C}$; umidade relativa média: 79,0 $\pm 10,3 \%$ e fotofase natural.

${ }^{2}$ Média não seguidas de mesma letra na coluna diferem entre si pelo teste de Tukey, em nível de 5\% de probabilidade de erro. 
O extrato a base de folhas de $\boldsymbol{S}$. foetida apresentou ação inseticida para ninfas de $\boldsymbol{B}$. tabaci. Este extrato ocasionou uma taxa de mortalidade de 75\%, diferindo estatisticamente dos tratamentos com sementes e folhas de $\mathbf{L}$. leucocephala e sementes de S. foetida, que apresentaram mortalidade variando de 18 a 28\%, não havendo, no entanto, diferença estatística entre estes três últimos tratamentos e a testemunha, que teve uma taxa de $8 \%$ de mortalidade (Tabela 1 ). SOUZA \& VENDRAMIM (2000) obtiveram taxa de mortalidade superior a esta quando trabalharam com extrato a 3\% de sementes de $A$. indica (86,6\%) e inferior para frutos verdes de $\boldsymbol{M}$. azedarach (54,9\%) e ramos de T. pallida (53,4\%). CAVALCANTE (2004) relata taxas de mortalidade para ninfa de $\boldsymbol{B}$. tabaci de 80,6\%; 75,1\% e $75,5 \%$, respectivamente, para extratos aquosos a $10 \%$ de folhas de A. indica, P. juliflora e L. leucocephala.

Índice de supressão de oviposição

Embora todos os tratamentos tenham diferido estatisticamente da testemunha, os índices de supressão de oviposição obtidos demonstram que os extratos testados não apresentam ação supressiva à oviposição de $\boldsymbol{B}$. tabaci, sendo classificados como neutros pela fórmula sugerida por KOGAN \& GOEDEN (1970) (Tabela 2).

\section{Longevidade e fertilidade}

Estatisticamente, todas as médias de longevidade diferiram entre si, tendo os tratamentos com L. leucocephala apresentado menor longevidade para machos e fêmeas em relação aos tratamentos com S. foetida e à testemunha (Tabela 3). Os indivíduos que foram submetidos ao tratamento com extrato aquoso de folhas de L. leucocephala viveram aproximadamente 3,5 dias a menos que os tratados com água destilada. O tratamento com sementes de $\boldsymbol{S}$. foetida apresentou a maior média de longevidade. Neste tratamento, os machos e as fêmeas viveram aproximadamente 3 e 4 dias, respectivamente, a mais que os adultos da testemunha. VILAS BÔAS et al. (2002) obtiveram valores para a longevidade de $\boldsymbol{B}$. tabaci que variaram de 12,9 a 21,3 dias em Brassica oleracea var. capitata.

A fecundidade de B. tabaci foi significativamente diferente para todos os tratamentos. As fêmeas dos tratamentos com L. leucocephala apresentaram as maiores médias de fecundidade e as tratadas com $\boldsymbol{S}$. foetida apresentaram as menores, sendo a testemunha um valor intermediário. VILAS BÔAS et al. (2002) obtiveram fecundidade variando entre 108,7 a 172,3 ovos por fêmea para $\boldsymbol{B}$. tabaci criada em $\boldsymbol{B}$. oleracea var. capitata. É provável que nos tratamentos com L. leucocephala tenha ocorrido um efeito de hormoligose, em que, segundo LUCKEY (1968), uma dose subletal do extrato testado tenha estimulado a oviposição na população submetida ao tratamento, podendo justificar-se desta forma o efeito inesperado do extrato, aumentando o número de ovos por fêmea em relação à testemunha.

\section{CONCLUSÃO}

Os extratos aquosos que demonstraram os melhores níveis de controle sobre $\boldsymbol{B}$. tabaci biótipo B (nas condições deste experimento) foram os de sementes de $\mathbf{L}$. leucocephala, quando aplicados na fase de ovo, e os de folhas de $\boldsymbol{S}$. foetida, quando aplicados nas fases de ovo e ninfa. Estes dois extratos apresentam boas perspectivas para serem utilizados no controle desse inseto em programas de manejo integrado de pragas.

Tabela 2 - Quantidade média de ovos por folha e índice médio de supressão de oviposição (ISO) para Bemisia tabaci biótipo B, tratadas com extratos aquosos a 10\% de Leucaena leucocephala, folha (F) e semente (S), e de Sterculia foetida, folha (F) e semente (S).

\begin{tabular}{|c|c|c|c|c|c|c|c|}
\hline \multirow{2}{*}{ Tratamento $^{1}$} & & \multicolumn{3}{|c|}{ Número de ovos / folha } & \multirow{2}{*}{ CV (\%) } & \multirow{2}{*}{$\mathrm{ISO} \pm \mathrm{DP}^{3}$} & \multirow{2}{*}{ Classificação } \\
\hline & & Tratamento & Testemunha & Média geral & & & \\
\hline \multirow{2}{*}{ L. leucocephala } & $\mathrm{F}$ & $26,0 \mathrm{~b}^{2}$ & $31,1 \mathrm{a}$ & 28,6 & 12,6 & $0,98 \pm 0,27$ & Neutro \\
\hline & $\mathrm{S}$ & 21,2 b & $21,7 \mathrm{a}$ & 21,5 & 1,65 & $0,94 \pm 0,20$ & Neutro \\
\hline \multirow{2}{*}{ S. foetida } & $\mathrm{F}$ & 30,8 a & $23,2 \mathrm{~b}$ & 27,0 & 19,9 & $1,05 \pm 0,23$ & Neutro \\
\hline & $\mathrm{S}$ & $23,1 \mathrm{a}$ & $22,6 \mathrm{~b}$ & 22,9 & 1,53 & $1,15 \pm 0,24$ & Neutro \\
\hline
\end{tabular}

${ }^{1}$ Temperatura média: $29,3 \pm 2,9^{\circ} \mathrm{C}$; umidade relativa média: $67 \pm 11,0 \%$ e fotofase natural.

${ }^{2}$ Médias não seguidas de mesma letra na linha, dentro de cada tratamento, diferem significativamente entre si pelo teste de Tukey em nível de $5 \%$ de probabilidade de erro.

${ }^{3}$ Desvio padrão. 
Tabela 3 - Longevidade, de machos e fêmeas, e fecundidade (médias \pm EP) de Bemisia tabaci biótipo B, tratadas com extratos aquosos a 10\% de Leucaena leucocephala e de Sterculia foetida.

\begin{tabular}{llccc}
\hline Tratamento $^{1}$ & Estrutura & Longevidade de machos (dias) & Longevidade de fêmeas (dias) & Fecundidade (ovos/fêmea) \\
\hline L. leucocephala & Folha & $17,4 \pm 2,14 \mathrm{e}^{2}$ & $17,4 \pm 1,65 \mathrm{e}$ & $95,3 \pm 8,82 \mathrm{~b}$ \\
& Semente & $18,1 \pm 2,19 \mathrm{~d}$ & $20,3 \pm 1,81 \mathrm{~d}$ & $100,0 \pm 2,06 \mathrm{a}$ \\
S. foetida & Folha & $19,8 \pm 3,12 \mathrm{c}$ & $20,4 \pm 1,70 \mathrm{c}$ & $58,6 \pm 5,55 \mathrm{e}$ \\
& Semente & $24,0 \pm 1,50 \mathrm{a}$ & $25,1 \pm 1,20 \mathrm{a}$ & $76,9 \pm 8,01 \mathrm{~d}$ \\
Testemunha & Água & $21,1 \pm 1,83 \mathrm{~b}$ & $20,9 \pm 2,57 \mathrm{~b}$ & $79,1 \pm 15,91 \mathrm{c}$ \\
Média geral & -- & $20,1 \pm 2,16$ & $22,8 \pm 1,79$ & $82,0 \pm 8,07$ \\
CV (\%) & -- & 14,22 & 11,38 & 13,69 \\
\hline
\end{tabular}

${ }^{1}$ Temperatura média: $30,0^{\circ} \mathrm{C} \pm 2,9$; umidade relativa média: $70,0 \% \pm 9,5$ e fotofase natural.

${ }^{2}$ Médias não seguidas pela mesma letra na coluna diferem entre si pelo teste de Tukey em nível de 5\% de probabilidade de erro.

\section{AGRADECIMENTOS}

Ao Conselho Nacional de Desenvolvimento Científico e Tecnológico (CNPq), pela concessão da bolsa de iniciação científica ao primeiro autor, e à Universidade Federal Rural de Pernambuco, por possibilitar a execução deste trabalho.

\section{REFERÊNCIAS}

AZEVEDO, J.A. et al. Eficiência de produtos naturais para o controle de Bemisia tabaci biótipo B (Hemiptera: Aleyrodidae) em meloeiro. Arquivos do Instituto Biológico, São Paulo, v.72, n.1, p.73-79, 2005.

BALDIN, E.L.L. et al. Resistência de genótipos de tomateiro à mosca-branca Bemisia tabaci (Gennadius) biótipo B (Hemiptera: Aleyrodidae). Neotropical Entomology, Londrina, v.34, n.3, p.435-441, 2005.

CAVAlcante, G.M. Potencial inceticida dos extratos aquosos de essências florestais arbóreas sobre a mosca branca Bemisia tabaci biótipo B (Gennadius, 1889) (Hemiptera: Aleyrodidae). 2004. 43f. Dissertação (Mestrado em Ciências Florestais) - Curso de Pós-graduação em Ciências Florestais, Universidade Federal Rural de Pernambuco.

FILGUEIRA, F.A.R. Novo manual de olericultura: agrotecnologia moderna na produção e comercialização de hortaliças. 2.ed. Viçosa: UFV, 2000. 402p.

GERLING, D. Whiteflies revisited. In: INTERNATIONAL CONGRESS OF ENTOMOLOGY, 21., 2000, Foz do Iguaçu, SC. Anais... Foz do Iguaçu: SEB, 2000. V.1. p.54-58.

HERNANDEZ, C.R.; VENDRAMIM, J.D. Uso de índices nutricionales para el efecto insectistatico de extratos de Meliaceas sobre Spodoptera frugiperda. Manejo Integrado de Plagas, Turrialba, n.48, p.11-18, 1998.

HAMMAD, E.M.A.F et al. Responses of the sweetpotato whitefly, Bemisia tabaci, to the chinaberry tree (Melia azedarach L.) and its extracts. Annals of Applied Biology, Warwick, v.137, n.2, p.79-88, 2000.

HOROWITZ, A.R.; ISHAAYA, I. Chemical control of Bemisia - management and application. In: GERLING, D.; MAYER,
R.T. Bemisia 1995: taxonomy, biology, damage, control and management. Andover: Intercept, 1995. p.537-556.

JACOBSON, M. Botanical pesticides: past, present and future. In: ARNASON, J.T. et al. Inseticides of plant origin. Washington: American Chemical Society, 1989. Cap.1, p.110 .

KOGAN, M.; GOEDEN, R.D. The host-plant range of Lema trilineata daturaphila (Coleoptera: Chrysomelidae). Annals of the Entomological Society of America, College Park, v.63, n.4, p.1175-1180, 1970.

LANCHER, W. Ecofisiologia vegetal. São Carlos: Rima, 2000. 519p.

LIMA, M.I.S. Substâncias do metabolismo secundário de algumas espécies nativas e introduzidas no Brasil. In: LANCHER, W. Ecofisiologia vegetal. São Carlos: Rima, 2000. p.33-68.

LUCKEY, T.D. Insecticide hormoligosis. Journal of Economic Entomology, Lanham, v.61, n.1, p.7-12, 1968.

MARTIN, J.H. An identification guide to common whitefly pest species of the world (Homoptera, Aleyrodidae). Tropical Pest Management, Basingstoke, v.33, n.4, p.298-322, 1987.

MEDEIROS, A.R.M. Alelopatia: importancia e suas aplicações. Hortisul, v.1, n.3, p.27-32, 1990.

NARDO, E.A.B. de et al. Melia azedarach extract as an antifeedant to Bemisia tabaci (Homoptera: Aleyrodidae). Florida Entomologist, Gainesville, v.80, n.1, p.92-94, 1997.

OLIVEIRA, M.R.V. et al. History, current status, and collaborative research projects for Bemisia tabaci. Crop Protection, Oxford, v.20, n.9, p.709-723, 2001.

PRATES, H.T. et al. Efeito do extrato aquoso de leucena na germinação e no desenvolvimento do milho. Pesquisa Agropecuária Brasileira, Brasília, v.35, n.5, p.909-914, 2000.

SANTOS, T.O. dos et al. Escarificação mecânica em sementes de chichá (Sterculia foetida L.). Revista Árvore, Viçosas-MG, v.28, n.1, p.1-6, 2004.

Ciência Rural, v.36, n.5, set-out, 2006. 
SILVA, L.D. da et al. Eficiência de azadiractina no controle de mosca-branca em meloeiro sob condições de casa de vegetação e de campo. Horticultura Brasileira, Brasília, v.21, n.2, p.198-201, 2003.

SKERMAN, P.J. Tropical forage legumes. Rome: FAO, 1977. 609p.

SOUZA, A.P. de. Atividade inseticida e modo de ação de extratos de meliáceas sobre Bemisia tabaci (Genn., 1889) biótipo B. 2004. 101f. Tese (Doutorado em Entomologia) Curso de Pós-graduação em Entomologia, Escola Superior de Agricultura "Luiz de Queiroz".

SOUZA, A.P. de; VENDRAMIM, J.D. Efeito de extratos aquosos de meliáceas sobre Bemisia tabaci biótipo B em tomateiro. Bragantia, Campinas, v.59, n.2, p.173-179, 2000.

SUJII, E.R. et al. Metodologia de amostragem de ninfas e avaliação preliminar de fungos entomopatogênicos contra a mosca branca no meloeiro. Brasília: EMBRAPA-
CENARGEN, 2002. 15p. (Boletim de pesquisa e desenvolvimento, 27)

TAVARES, M.A.G.C. Bioatividade da erva-de-santa-maria, Chenopodium ambrosioides L. (Chenopodiaceae), em relação a Sitophilus zeamais Mots., 1855 (Col.: Curculionidae). 2002. 59f. Dissertação (Mestrado em Entomologia) - Curso de Pós-graduação em Entomologia, Escola Superior de Agricultura "Luiz de Queiroz".

TOSCANO, L.C. et al. Preferência de Bemisia tabaci biótipo B para oviposição em cultivares de algodoeiro. Pesquisa Agropecuária Brasileira, Brasília, v.38, n.1, p.155-160, 2003.

VILLAS BÔAS, G.L. et al. Manejo Integrado da mascabranca Bemisia argentifolii. Brasília: EMBRAPA-CNPH, 1997. 12p. (Circular Técnico, 9).

VILLAS BÔAS, G.L. et al. Potencial biótico da mosca-branca Bemisia argentifolii a diferentes plantas hospedeiras. Horticultura Brasileira, Brasília, v.20, n.1, p.71-79, 2002. 